\title{
DIVERSIDAD GENÉTICA DE MARACUYÁ EN GUATEMALA REVELADA POR MARCADORES AFLP ${ }^{1}$
}

\author{
Karla Melina Ponciano-Samayoa ${ }^{2}$, Juan Pedro Lacán de León ${ }^{2}$
}

\section{RESUMEN}

Diversidad genética de maracuyá en Guatemala revelada por marcadores AFLP. Los objetivos de este estudio fueron caracterizar con AFLP nueve genotipos colectados en Guatemala y determinar la diversidad genética existente. En el Instituto de Ciencia y Tecnología Agrícolas (ICTA), en el período julio 2010/mayo 2011, se realizó este análisis preliminar amplificando diez combinaciones selectivas con las que se detectaron 106 polimorfismos. Las combinaciones selectivas $\mathrm{E}+\mathrm{ACG} / \mathrm{M}+\mathrm{CAG}, \mathrm{E}+\mathrm{ACA} / \mathrm{M}+\mathrm{CTA}$, $\mathrm{E}+\mathrm{ACT} / \mathrm{M}+\mathrm{CTG}$ y $\mathrm{E}+\mathrm{AAC} / \mathrm{M}+\mathrm{CTT}$ dieron el mayor grado de información. En promedio se visualizaron diez loci por amplificación selectiva. El análisis de similaridad reveló que los genotipos no están duplicados. Los análisis de correspondencia y conglomerados identificaron dos grupos bien definidos. El primero incluyó a los materiales de $P$. edulis $\mathrm{f}$. edulis Sims y el segundo a los materiales de P.edulis f. flavicarpa Degener. La diversidad genética de Nei para la colección fue 0,3160. La diferenciación genética $\left(\mathrm{G}_{\mathrm{st}}\right)$ fue 0,2542 . El 25,42\% de la diversidad se expresó entre grupos mientras que el 74,58\% dentro de estos. Los resultados dan evidencia de la cercanía evolutiva de los tipos amarilla y morada de $P$. edulis Sims. El flujo genético fue alto $(\mathrm{Nm}=1,4670)$ como se esperaba en una especie alógama en la que se favorece el intercambio inter e intraespecífico.

Palabras clave: Passiflora edulis Sims, similaridad, diferenciación genética, conglomerados, flujo genético.

\begin{abstract}
Genetic diversity of passion fruits in Guatemala revealed by AFLP markers. The objectives of this study were to characterize with AFLP nine genotypes of passion fruit collected in Guatemala, to identify clusters and to evaluate the existing genetic diversity. The study was conducted at Instituto de Ciencia y Tecnología Agrícolas (ICTA), during July 2010/May 2011. A preliminary analysis was conducted in which ten selective primer pairs were amplified and 106 polymorphisms were detected. The selective primer pair $\mathrm{E}+\mathrm{ACG} / \mathrm{M}+\mathrm{CAG}, \mathrm{E}+\mathrm{ACA} / \mathrm{M}+\mathrm{CTA}, \mathrm{E}+\mathrm{ACT} / \mathrm{M}+\mathrm{CTG}$ and $\mathrm{E}+\mathrm{AAC} / \mathrm{M}+\mathrm{CTT}$, gave the most information. An average of ten loci per selective amplification was detected. Similarity analysis revealed no duplicated genotypes. Correspondence and clustering analysis identified two groups. The first one included all accession of $P$. edulis f. edulis Sims and the second one all accessions of $P$. edulis f. flavicarpa Degener. The genetic diversity based on Nei's index for the collection was 0,3160 . Genetic differentiation $\left(\mathrm{G}_{\mathrm{st}}\right.$ was 0,2542 for the entire collection; $25.42 \%$ of the genetic diversity is expressed between groups while the $74,58 \%$ is expressed within groups. Those results give evidence of the evolutionary proximity of the purple and yellow types of P. edulis Sims. The genetic flow was high $(\mathrm{Nm}=1,4670)$ as expected in an allogamous species in which genetic exchange is facilitated.

Key words: Passiflora edulis Sims, similarity, genetic differentiation, clusters, genetic flow.
\end{abstract}

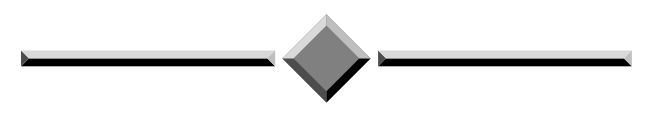

\footnotetext{
1 Recibido: 28 de junio, 2011. Aceptado: 12 de marzo, 2012. Proyecto de investigación del Laboratorio de Biotecnología y el Programa de Promoción y Apoyo Tecnológico. Instituto de Ciencia y Tecnología Agrícolas ICTA.

2 Laboratorio de Biotecnología y Programa de Promoción y Apoyo Tecnológico. Instituto de Ciencia y Tecnología Agrícolas ICTA. Km. 21.5 Carretera al Pacífico, Bárcenas, Villa Nueva, Guatemala, C.A. kponciano@hotmail.com; lacandeleon@yahoo.com
} 


\section{INTRODUCCIÓN}

El maracuyá (Passiflora edulis Sims) es una especie neotropical perteneciente a la familia Passifloraceae. Tuvo su origen en Brasil y durante el siglo XIX se extendió a otros países debido a su alto potencial agrícola y de exportación gracias a su buena adaptabilidad a nuevos ambientes. Sus cualidades más apreciadas son su sabor, apariencia, valor nutritivo, disponibilidad, accesibilidad, propiedades medicinales y connotación exótica (Fonseca et al. 2009). También, se ha reportado que los residuos del fruto de maracuyá tienen alta capacidad de absorción de arsénico y pueden ser útiles en los programas de remediación ambiental (Iliná et al. 2009).

$P$. edulis es una especie predominantemente alógama que presenta un sistema genético de autoincompatibilidad que favorece la polinización cruzada inter e intra-específica (Fonseca et al. 2009). Se ha reportado que esta especie presenta un $80 \%$ de autoincompatibilidad, por lo tanto un $20 \%$ de autopolinización (Ortiz 2010). Los estudios citogenéticos indican que es una especie diploide $(2 \mathrm{n}=18)$ (Crochemore et al. 2003). Se conocen dos formas de $P$. edulis que se diferencian principalmente en el color del fruto, el aroma y el sabor. P. edulis f. edulis Sims es conocida como maracuyá morado (Crochemore et al. 2003), gulupa, purple passion fruit (Ortiz 2010) o maracujá-roxo (Sousa et al. 1997). Por otro lado, P. edulis f.flavicarpa Degener se conoce como maracuyá amarillo (Crochemore et al. 2003), simplemente maracuyá, yellow passion fruit (Ortiz 2010) o maracujá-amarelo (Sousa et al. 1997). El maracuyá amarillo es la forma más conocida en el comercio agrícola pero su producción ha disminuido, debido a la competencia y altos precios, dando lugar al maracuyá morado como alternativa más agradable al consumidor debido a su aroma más fuerte y sabor más dulce respecto al fruto amarillo (Ortiz 2010).

A parte del color del fruto, el sabor y la resistencia a enfermedades, $P$. edulis f. edulis y P. edulis f. flavicarpa no muestran diferencias contrastantes a simple vista (Crochemore et al. 2003). Sin embargo, la caracterización molecular puede diferenciar entre individuos de $P$. edulis sin dependencia del ambiente (Fonseca et al. 2009).

La mayoría de los estudios moleculares han reportado el uso exitoso de las técnicas RAPD, AFLP y SSR para establecer la diversidad genética entre y dentro de especies silvestres y comerciales de Passiflora ssp., colectadas en países como Brasil, Colombia, Australia y Venezuela (Andrade et al. 2002, Crochemore et al. 2003, Fonseca et al. 2009, Oliveira et al. 2005, Ortiz 2010, Pérez et al. 2009). La mayoría de las investigaciones se ha hecho con RAPD, sin embargo se considera que AFLP es un mejor sistema para generar huellas de $\mathrm{ADN}$ por ser altamente reproducibles y no requiere de conocimiento previo de la secuencia de ADN (Tang et al. 2003, Ganga et al. 2004.).

Andrade et al. (2002) establecieron que $P$. edulis f. edulis Sims y su variedad botánica P. edulis f. flavicarpa Degener tienen una similaridad del $34.35 \%$ por lo que son altamente variables cuando son analizados con RAPDs. Más interesantes aún fueron los resultados reportados por Crochemore et al. (2003) pues revelaron evidencia de que $P$. edulis f. edulis es ascendente genético de $P$. edulis f. flavicarpa y que la segunda se diferencia dentro del grupo de la primera. En otras palabras, que la forma amarilla se originó a partir de una mutación de $P$. edulis f. edulis y no de un cruce interespecífico con una especie diferente.

Tanto Oliveira et al. (2005) como Fonseca et al. (2009) introdujeron el uso de microsatélites nuevos y al azar para la determinación de la diversidad genética entre materiales de la misma forma genética.

Las técnicas AFLP y SSR fueron utilizadas (Ortiz 2010) para estudiar la diversidad genética inter-específica en materiales de maracuyá amarillo colectados en Colombia, mostrando poca variabilidad entre ellos. Sin embargo, sí encontró ventajas en la técnica AFLP para discriminar al maracuyá amarillo del morado. En este caso, los SSR reportados por Oliveira et al. (2005) no fueron informativos.

En términos generales, se ha establecido que el índice de diversidad genética de $P$. edulis Sims es alto $\mathrm{y}$ debe estar dado por el flujo de genes inter e intraespecífico (Fonseca et al. 2009) en cruces naturales $\mathrm{y}$ artificiales entre individuos adaptados a diferentes regiones agro-climáticas (Crochemore et al. 2003).

En Guatemala, el cultivo de maracuyá es una introducción reciente que ya muestra diversidad agromorfológica y potencial económico para pequeños productores. Debido a esto, el ICTA realizó un muestreo en jardines de productores que usan variedades comerciales introducidas de otros países y el resultado fue la primera colección guatemalteca de $P$. edulis que agrupa a nueve genotipos (Lacán e Illescas 2010). 
El estudio de diversidad genética de dicha colección generaría información útil para discriminar entre las introducciones de P. edulis f. edulis y P. edulis f. flavicarpa, detectar duplicados y establecer a mediano plazo programas de mejoramiento genético, agroindustria, conservación, diversificación de cultivos y alimentos para consumo nacional y exportación.

El objetivo de este trabajo fue caracterizar, con marcadores moleculares AFLPs, nueve materiales de maracuyá cultivados en Guatemala para determinar la diversidad genética existente.

\section{MATERIALES Y MÉTODOS}

Material vegetal. En el período julio 2010/mayo 2011, se analizaron nueve accesiones de maracuyá colectadas en un estudio anterior en campos productores de Guatemala (Lacán e Illescas 2010). La caracterización morfológica de dichos materiales no reveló la diversidad genética presente. El avance más significativo fue determinar a qué forma botánica pertenecen las accesiones colectadas, utilizando el color del fruto, el aroma y el sabor como marcadores. De aquí que los nueve materiales colectados se clasificaran en cinco pertenecientes a las especies $P$. edulis f. edulis, de fruto morado, y cuatro de P. edulis f. flavicarpa, de fruto amarillo (Cuadro 1) (Lacán e Illescas 2010). Para este estudio molecular se ordenaron según su clasificación varietal y se conservó el número de accesión correspondiente a cada material en la colección (Cuadro 1).
Extracción, verificación de integridad y cuantificación de ADN. Las primeras hojas, diferentes de las primarias, de tres plantitas de quince días de edad, fueron seleccionadas al azar para hacer una mezcla de material vegetal (aproximadamente $200 \mathrm{mg}$ ), la cual fue macerada con pistilos y nitrógeno líquido dentro de tubos de microcentrífuga de 1,5 ul. El polvo obtenido se sometió al protocolo de extracción propuesto como se describe en Ponciano et al. 2009. El precipitado resultante se resuspendió en $150 \mu \mathrm{l}$ de buffer TE (10mM Tris-HCL, 1mM EDTA, pH 8,00) y se almacenó a $4^{\circ} \mathrm{C}$. La integridad del ADN fue verificada cargando $10 \mu \mathrm{l}$ en un gel de agarosa al 0,8\%. A partir de diluciones 1:200 se cuantificó el ADN en un espectrofotómetro Bio-Rad SmartSpec3000 con un factor de conversión A260 nm 1,0=50,0 $\mu \mathrm{g} / \mathrm{ml}$. La calidad del ADN se determinó a partir de la razón A260/A280. El ADN se diluyó a $25 \mathrm{ng} / \mu \mathrm{l}$ para las siguientes amplificaciones (Ponciano 2004).

Amplificación y visualización de AFLPs. Para este primer intento de establecer la huella genética de las accesiones de maracuyá, se propuso la técnica AFLP para generar información sobre similitudes y diferencias entre los materiales con base en los alelos presentes para un rango de loci amplificados a lo largo del genoma. Los recursos disponibles limitaron el estudio a la amplificación de diez combinaciones de partidores selectivos (Cuadro 2). Estas fueron escogidas con base en información reportada previamente en amplificación de RAPDs, RAM y SSR (Andrade et al. 2002, Crochemore et al. 2003, Fonseca et al. 2009,

Cuadro 1. Identificación de las accesiones caracterizadas con AFLP (Lacán e Illescas 2010). Guatemala, enero 2010.

\begin{tabular}{ccccc}
\hline Accesión & Especie & Color de fruto & Grados Brix, jugo & Lugar de colecta \\
\hline C1(1) & P. edulis f. edulis Sims & Morado & 15 & Totonicapán, Guatemala \\
C2(2) & P. edulis f. edulis Sims & Morado & 15 & Guatemala, Guatemala \\
C3(8) & P. edulis f. edulis Sims & Morado & 16 & Sacatepéquez, Guatemala \\
C4(9) & P. edulis f. edulis Sims & Morado & 18 & Sacatepéquez, Guatemala \\
C5(10) & P. edulis f. edulis Sims & Morado & 17 & Chimaltenango, Guatemala \\
C6(3) & P. edulis f. flavicarpa Deg. & Amarillo & 14 & Zamorano, Honduras \\
C7(5) & P. edulis f. flavicarpa Deg. & Amarillo & 14 & Guatemala, Guatemala \\
C8(6) & P. edulis f. flavicarpa Deg. & Amarillo & 11 & Huehuetenango, Guatemala \\
C9(7) & P. edulis f. flavicarpa Deg. & Amarillo & 15 & Huehuetenango, Guatemala \\
\hline
\end{tabular}


Cuadro 2. Amplificaciones selectivas utilizadas en la caracterización molecular de maracuyá y número de polimorfismo AFLP revelados. Guatemala, diciembre 2009.

\begin{tabular}{ccc}
\hline & Combinación AFLP & No. bandas polimórficas \\
\hline 1 & E+ACA /M+CAA & 08 \\
2 & E+ACC /M+CAC & 02 \\
3 & E+ACG /M+CAG & 28 \\
4 & E+ACT /M+CAT & 02 \\
5 & E+ACA /M+CTA & 16 \\
6 & E+AGC /M+CTA & 08 \\
7 & E+AGG /M+CTC & 00 \\
8 & E+ACT /M+CTG & 23 \\
9 & E+AAC /M+CTT & 11 \\
10 & E+ACA /M+CTT & 08 \\
\hline & Total & $\mathbf{1 0 6}$ \\
\hline
\end{tabular}

Oliveira et al. 2005) sobre secuencias de nucleótidos más frecuentes en maracuyá.

Para todas las muestras se amplificaron marcadores AFLP utilizando el sistema AFLP ${ }^{\circledR}$ Analysis System I (Invitrogen ${ }^{\mathrm{TM}}$ Life Technologies, California, USA) (Invitrogen Corporation 2003) siguiendo los protocolos establecidos por el proveedor. Se utilizó una alícuota de $10 \mu 1$ de ADN genómico total diluido en la reacción de digestión con las enzimas de restricción EcoR I y Mse I. Las reacciones se incubaron a $37^{\circ} \mathrm{C}$ por dos horas, seguido de una incubación a $70^{\circ} \mathrm{C}$ por quince minutos y una incubación en hielo por cinco minutos para desactivar las enzimas. Los fragmentos de ligación resultantes fueron ligados a adaptadores EcoR I y Mse I para generar el ADN molde el cual fue utilizado en las amplificaciones siguientes. $\mathrm{La}$ preamplificación consistió en amplificar el ADN molde con partidores complementarios a los adaptadores y al sitio de restricción Mse I más un nucleótido adenina (Partidor Mse I +A) y al sitio de restricción EcoR I más un nucleótido citosina (Partidor EcoR I $+\mathrm{C}$ ). El programa de preamplificación consistió en 20 ciclos de desnaturalización a $94^{\circ} \mathrm{C}$ por 30 segundos, seguido de hibridización a $56^{\circ} \mathrm{C}$ por 60 segundos y una elongación a $72^{\circ} \mathrm{C}$ por 60 segundos. La preamplificación de las muestras fue verificada cargando $10 \mu 1$ de producto de PCR en un gel de agarosa 1,2\%. Las muestras se mezclaron con buffer de carga y corridas por $30 \mathrm{mi}-$ nutos a $50 \mathrm{~V}$ y el gel se tiñó con bromuro de etidio. En todas las muestras, el producto de preamplificación fue amplificado selectivamente utilizando combinaciones de partidores EcoR I y Mse I más tres nucleótidos selectivos adicionales cada uno (Cuadro 2).

Estos marcadores fueron amplificados por medio de PCR convencional en placas de 96 pozos en un termociclador ATC401 Apollo CLP. El programa de amplificación selectiva consistió en 36 ciclos distribuidos en dos etapas: Etapa 1. Trece ciclos de desnaturalización a $94^{\circ} \mathrm{C}$ por 30 segundos, seguido de hibridización a $65^{\circ} \mathrm{C}$ por 60 segundos y una elongación a $72^{\circ} \mathrm{C}$ por 60 segundos; con una reducción de $0,7^{\circ} \mathrm{C}$ en la temperatura de hibridización por cada ciclo sucesivo. Etapa 2. Veintitrés ciclos de desnaturalización a $94^{\circ} \mathrm{C}$ por 30 segundos, seguido de hibridización a $56^{\circ} \mathrm{C}$ por 60 segundos y elongación a $72^{\circ} \mathrm{C}$ por 60 segundos. Las mezclas de reacción finales fueron preparadas según las cantidades indicadas en el protocolo del proveedor (Invitrogen Corporation 2003).

Después de la amplificación, se agregó un volumen igual de solución de formamida al $95 \%$ formamida, $3 \%$ agua, $2 \%$ EDTA0.5M, $0,01 \%$ azul de bromofenol y $0,01 \%$ xilen cianol) y la mezcla fue desnaturalizada por tres minutos a $94^{\circ} \mathrm{C}$ y durante dos minutos en hielo. Se realizó una electroforesis vertical en gel de poliacrilamida al 5\% (acrilamida:bis-acrilamida 29:1) en condiciones desnaturalizantes con $5 \mathrm{M}$ de urea en $1 X$ TBE. Se corrieron en unidades de electroforesis Bio-Rad Sequi-Gen GT a una potencia constante de $50 \mathrm{~W}$ durante una hora quince minutos. En todos los geles se incluyó una escalera de peso molecular de 100 pares de bases (pb). El procedimiento de tinción se realizó como se describe en Ponciano et al. (2009). El gel se secó por veinticuatro horas y se obtuvo una imagen por scanner (Ponciano et al. 2009).

Análisis de datos. A partir de los patrones de bandas visualizadas en los geles, se codificó la información alélica en una matriz de ausencia y presencia, la cual fue utilizada para análisis de similaridad y de correspondencia en NTSYS-pc 2.02c (Rohlf 1992). Se utilizó el subprograma SIMQUAL para crear una matriz de distancias genéticas con coeficiente de Dice. Esta matriz se utilizó en el subprograma SAHN para realizar un análisis de conglomerados y construir un dendrograma basado en el método de agrupamiento UPGMA y visualizarlos con el subprograma TREE 
PLOT. Con el subprograma CORRESP se construyó un diagrama en dos dimensiones mostrando la distribución de las accesiones en el espacio (Ponciano et al. 2009). La diversidad genética entre los grupos identificados en el dendrograma basada en distancias genéticas de Nei (Nei 1978) se calculó utilizando el programa POPGENE versión 1.32 (Yeh et al. 1997). La matriz utilizada para este programa se construyó representando cada banda o alelo amplificado como un genotipo diferente dada la dominancia de los AFLPs (Wagara et al. 2004). El análisis estadístico se realizó en los módulos DOMINANT DATA ANALYSIS- DIPLOID DATA-SINGLE POPULATIONS AND MULTIPLE POPULATIONS. Los parámetros de diversidad genética determinados fueron: distancia genética de Nei (Nei), índice de diferenciación genética $\left(\mathrm{G}_{\mathrm{ST}}\right)$, y flujo genético $(\mathrm{Nm})$. La diferenciación genética fue calculada con la formula de Nei (1978) $\mathrm{G}_{\mathrm{ST}}=\mathrm{D}_{\mathrm{ST}} /$ $\mathrm{H}_{\mathrm{T}}$ donde $\mathrm{H}_{\mathrm{T}}=\mathrm{H}_{\mathrm{S}}+\mathrm{D}_{\mathrm{ST}}$ y $\mathrm{D}_{\mathrm{ST}}=\mathrm{H}_{\mathrm{T}}-\mathrm{H}_{\mathrm{S}}$, donde $\mathrm{H}_{\mathrm{T}}$ es la diversidad total para la poblaciones y $\mathrm{D}_{\mathrm{ST}}$ es la diversidad genética entre poblaciones (Blair et al. 2007). El flujo genético es igual al número de migrantes entre poblaciones por generación calculada en base a la fórmula $\mathrm{Nm}=0,5\left(1-\mathrm{G}_{\mathrm{ST}}\right) / \mathrm{G}_{\mathrm{ST}}$ (Blair et al. 2007).

\section{RESULTADOS Y DISCUSIÓN}

\section{Análisis de AFLPs en la colección de maracuyá}

Se recopiló la información de un total de 106 bandas polimórficas, que fueron tratadas como loci individuales debido a la naturaleza dominante de los marcadores AFLPs (Cuadro 2). Todas las bandas monomórficas fueron eliminadas del análisis. El promedio fue de diez AFLPs por combinación selectiva. Comparado con el promedio de veintiocho bandas polimórficas por combinación, reportado previamente (Ortiz 2010), es un valor bajo. La diferencia puede estar dada porque no se incluyeron genotipos nativos o de otras especies cercanas dentro de los genotipos comerciales de Guatemala evaluados en este estudio. Lamentablemente, los resultados del estudio de Ortiz (2010) fueron publicados después de que se realizaran las amplificaciones selectivas en este trabajo por lo que no se pudieron tener en cuenta en esta caracterización.

El rango de bandas polimórficas amplificadas fue de 0 a 28. La combinación E+ACG/M+CAG fue la más informativa con veintiocho loci polimórficos y la menos informativa fue $\mathrm{E}+\mathrm{AGG} / \mathrm{M}+\mathrm{CTC}$ con cero loci amplificados. Esta combinación fue eliminada del análisis estadístico. El número de AFLPs amplificados depende del cultivo que se trabaja por lo que es recomendable amplificar todas las combinaciones posibles y utilizar aquellas que amplifiquen más bandas polimórficas. La información generada con las combinaciones usadas en esta investigación puede ser de utilidad para estudios futuros pues complementan las combinaciones recomendadas por estudios previos como el de Ganga et al. (2004) y Ortiz (2010). Aunque es deseable amplificar más de diez combinaciones para establecer de manera concluyente la diversidad genética presente en una población, este estudio se puede considerar como preliminar y muy informativo ya que no se tiene más información del contenido genético de esta colección de maracuyá.

La accesión 5(10) no amplificó AFLPs en las combinaciones nueve y diez.

\section{Análisis de similaridad genética y agrupamientos}

Para establecer la diversidad genética a partir de los datos generados en la visualización de fragmentos polimórficos se construyó una matriz de similaridad con el coeficiente de Dice (1945). En la representación gráfica de la relación genética entre genotipos o dendrograma UPGMA (Figura 1) y se evidenció que todos los materiales son genéticamente diferentes. Así se descartó la sospecha de genotipos duplicados (Crochemore et al. 2003). El análisis de agrupamientos del dendrograma identificó dos grupos con un 44\% de similitud. La accesión C2(2) se separa del resto con un $40 \%$ de similaridad. Este resultado puede interpretarse como la posibilidad de que dicho material esté emparentado con un genotipo más ancestral y común al resto de accesiones. La accesión C5(10) fue eliminada del análisis por falta de datos.

La primera población incluye a los materiales pertenecientes a la especie $P$. edulis f. edulis Sims. Si se cambia la posición espacial de la accesión C2(2) hacia arriba en el dendrograma, se podría agrupar junto a esta población dando sentido al resultado previo en que fue clasificada morfológicamente como $P$. edulis forma edulis (Cuadro 1). El análisis de correspondencia (Figura 2) apoya la acción de incluir el genotipo C2 (2) en la agrupación, ya que la representación espacial en 


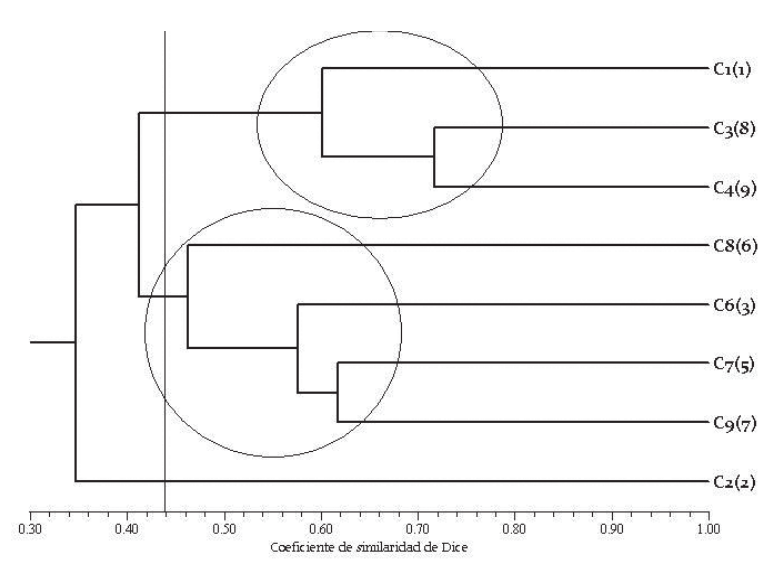

Figura 1. Dendrograma de la relación entre las muestras de la colección de maracuyá basada en el coeficiente de similaridad de Dice para nueve combinaciones selectivas de AFLP. Guatemala, mayo 2011.

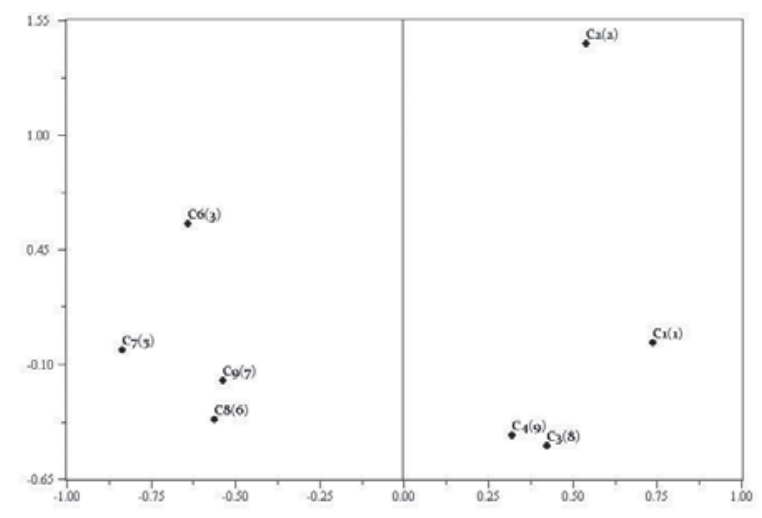

Figura 2. Análisis de correspondencia en dos dimensiones de la colección de maracuyá. Guatemala, mayo 2011.

dos dimensiones muestra su cercanía al conglomerado de genotipos $\mathrm{C} 1(1), \mathrm{C} 3(8)$ y $\mathrm{C} 4(9)$. El hecho de que se separe puede ser debido a que es una introducción proveniente de Brasil que tiene menos tiempo de establecimiento en Guatemala y por lo mismo tenga un grado mayor de diferenciación respecto al resto de genotipos en la colección (Fonseca et al. 2009). Por otro lado, los genotipos C3(8) y C4(9), que fueron colectados en la misma finca en Sacatepéquez, aunque son diferentes tienen una similitud del $74 \%$. Estos pueden ser producto de la polinización cruzada entre estas accesiones colectadas en ubicaciones tan cercanas.
La segunda población agrupa a todos los genotipos identificados como P. edulis f. flavicarpa. Con estos resultados se corrobora la clasificación inicial de los materiales colectados respecto a su inclusión en una u otra forma de maracuyá principalmente por el color de fruto. Dicha característica es un buen marcador para la clasificación de nuevos genotipos (Sousa y Meletti 1997). Las accesiones C8(6) y C9(7) fueron colectados en el mismo municipio de Huehuetenango pero solo comparten un coeficiente de similaridad de 0,46.

Un nuevo análisis de similaridad fue realizado entre las accesiones clasificadas dentro de la especie P. edulis f. edulis para establecer la relación entre la accesión C5(10), que inicialmente fue excluida, y el resto de materiales de fruto morado. Aquí se utilizó la información genotípica generada con las combinaciones selectivas donde el material C5(10) amplificó en la misma proporción que el resto. El dendrograma resultante (Figura 3 ) es consistente con la agrupación de la colección completa ya que el genotipo 2 queda fuera del conglomerado de los genotipos C1(1), C3(8) y C4(9). Dentro de este conjunto mayor se agrupa el material C5(10) con un coeficiente de similaridad de 0,53.

\section{Análisis de la diversidad genética}

La diversidad genética calculada por el índice de Nei para todos los genotipos de la colección de maracuyá fue 0,3160 (Cuadro 3). Se observó que el grupo de $P$. edulis f. edulis presentó el mayor valor de diversidad $(0,2725)$.

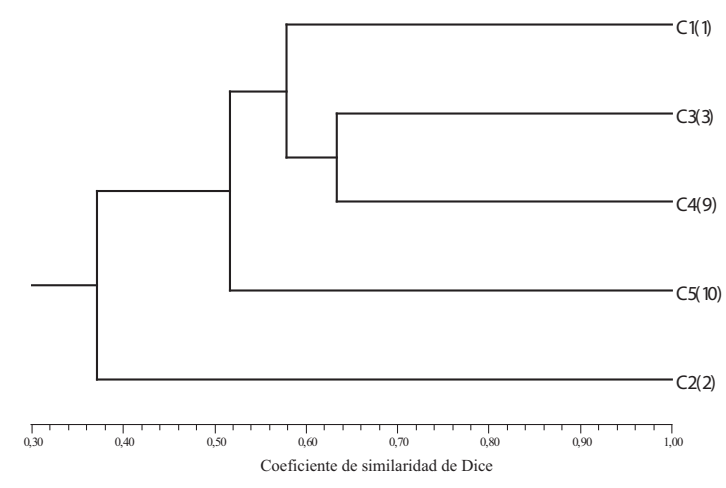

Figura 3. Dendrograma de la relación entre los genotipos de P. edulis f. edulis basada en el coeficiente de similaridad de Dice para seis combinaciones selectivas de AFLP. Guatemala, mayo 2011. 
Cuadro 3. Parámetro de diversidad genética para las poblaciones identificadas en el análisis de agrupamientos. Guatemala, mayo 2011.

\begin{tabular}{lccccc}
\hline Población & Nei & $\mathbf{H}_{\mathbf{t}}$ & $\mathbf{H}_{\mathbf{s}}$ & $\mathbf{G}_{\mathrm{st}}$ & $\mathbf{N m}$ \\
\hline 1. P. edulis f. edulis & 0,2725 & & & & \\
2. P. edulis f. flavicarpa & 0,1988 & & & & \\
\hline Total & 0,3160 & 0,3160 & 0,2357 & 0,2542 & 1,4670 \\
\hline
\end{tabular}

Abreviaciones: Diversidad genética según Nei (1978), diversidad total $\left(\mathrm{H}_{\mathrm{T}}\right)$, diversidad entre grupos $\left(\mathrm{H}_{\mathrm{s}}\right.$ ) , diferenciación genética $\left(\mathrm{G}_{\mathrm{st}}\right)$, flujo genético $(\mathrm{Nm})$.

La diversidad genética entre las dos poblaciones, formadas a partir del análisis de conglomerados, relativa a la diversidad genética total fue 0,2542 . Este valor indica que hubo una baja diferenciación entre los grupos analizados, puesto que sólo el $25,42 \%$ de la variación genética observada en este estudio fue debida a la diferenciación entre las dos formas de maracuyá, comparada con el 74,58\% de variación observada dentro de los grupos. Esto apoya las diferencias genéticas establecidas previamente entre $P$. edulis f. edulis Sims y P. edulis f. flavicarpa Degener, para separarlas en grupos diferentes. También, el valor de diversidad genética dentro de las poblaciones hace evidente el alto grado de intercambio genético que hay entre los individuos (Ganga et al. 2004, Fonseca et al. 2009).

El flujo genético total fue alto (mayor de 1,0000) $(\mathrm{Nm}=1,4670)$ como se esperaba debido a la naturaleza alógama del maracuyá, en la cual se favorecen los cruzamientos entre los individuos del mismo material, entre estos de la misma forma botánica de maracuyá y entre individuos de diferentes especies. Esto coincide con resultados previos (Andrade et al. 2002, Crochemore et al. 2003, Ganga et al. 2004, Fonseca et al. 2009, Oliveira et al. 2005, Ortiz 2010).

La caracterización preliminar con AFLPs reveló alta diversidad natural dentro de la colección de maracuyá cultivado en Guatemala. No se detectaron genotipos duplicados y se observó que los materiales forman conglomerados de acuerdo a su forma botánica. De acuerdo a los valores de los parámetros calculados, la diferenciación genética entre agrupaciones fue alta y cada grupo mostró un amplio índice de diversidad.

Se determinó utilizar siete de las combinaciones AFLP reportadas en este estudio ( $\mathrm{E}+\mathrm{ACA} / \mathrm{M}+\mathrm{CAA}$, $\mathrm{E}+\mathrm{ACG} / \mathrm{M}+\mathrm{CAG}, \mathrm{E}+\mathrm{ACA} / \mathrm{M}+\mathrm{CTA}, \mathrm{E}+\mathrm{AGC} /$ $\mathrm{M}+\mathrm{CTA}, \mathrm{E}+\mathrm{ACT} / \mathrm{M}+\mathrm{CTG}, \mathrm{E}+\mathrm{AAC} / \mathrm{M}+\mathrm{CTT}, \mathrm{E}+\mathrm{ACA}$
(M+CTT). En el futuro, se debe amplificar otras combinaciones para establecer el grado de información genética que puede proporcionar cada una. Con la utilización de un mayor número de combinaciones selectivas, se pueden obtener resultados más concluyentes en nuevos estudios de diversidad genética tanto de maracuyá como de otros cultivos que empiecen a tener importancia económica en la región hispanoamericana.

\section{LITERATURA CITADA}

Andrade, A; De Macedo, E; Oliveira, J. 2002. Genetic variations among passion fruit species using RAPD markers. Revista Brasileira de Fruticultura 24(3):738-740.

Blair, M; Diaz, J; Hidalgo, R; Diaz, L; Duque, M. 2007. Microsatellite characterization of Andean races of common bean (Phaseolus vulgaris L.). Theoretical Applied Genetics 116:29-43.

Crochemore, M; Correa, H; Estevez, L. 2003. Genetic Diversity in Passion Fruit (Passiflora spp.) evaluated by RAPD markers. Brazilian Archives of Biology and Technology 46(4):521-527.

Dice, L. 1945. Measures of the amount of ecologic association between species. Ecology 26:297-302.

Fonseca, N; Marquez, P; Moreno, J; Teran, W; Schuler, I. 2009. Caracterización molecular de materiales cultivados de gulupa (Passiflora edulis f. edulis). Universitas Scientiarum 14(2-3):135-140.

Ganga, R; Ruggiero, C; Lemos, E; Grili, G; Gonçalves, M; Chagas, E; Wickert, E. 2004. Diversidade genética em maracujazeiro-amarelo utilizando marcadores moleculares fAFLP. Revista Brasileira de Fruticultura 26(3):494-498.

Iliná, A; Martinez, J; Segura, E; Villareal, J; Gregorio, K. 2009. Biosorción de arsénico en materiales derivados 
de maracuyá. Revista Internacional de Contaminación Ambiental 25(4):201-216.

Invitrogen Corporation. 2003. AFLP Analysis System I. Version B. User Manuel. USA. 18 p.

Lacan, J; Illescas, V. 2010. Evaluación de genotipos locales e introducidos de maracuyá (Passiflora spp.), enfatizando el aprovechamiento de frutales nativos. AGROCYT 026-2004. Guatemala, Guatemala. ICTASENACYT. 75 p.

Nei, M. 1978. Estimation of average heterozygocity and genetic distance from a small number of individuals. Genetics 89:583-590.

Oliveira, E; Padua, J; Zucchi, M; Camargo, L; Fungaro, M; Vieira, M. 2005. Development and characterization of microsatellites markers from yellow passion fruit (Passiflora edulis f. flavicarpa). Molecular Ecology Notes 5(2):331-333.

Ortiz, D. 2010. Estudio de la variabilidad genética en materiales comerciales de gulupa (Passiflora edulis $\mathrm{f}$. edulis Sims) en Colombia. Tesis Mag. Sc. Bogotá, Colombia. UNC. 127 p.

Perez, I; Vasquez, S; Perez, D; De La Rosa, O; Salazar, E. 2009. Huella genética de genotipos silvestres y comerciales de Passiflora spp. utilizando patrones RAPD. Bioagro 21(3):203-208.
Ponciano, K. 2004. Evaluación de técnicas de extracción de ADN y de visualización para marcadores microsatélites en caña de azúcar (Saccharum officinarum L.). Tesis de Licenciatura. Guatemala, Guatemala. UVG.63 p.

Ponciano, K; Villatoro, J; Molina, L. 2009. Caracterización preliminar con microsatélites de la colección guatemalteca de frijol trepador. Agronomía Mesoamericana 20(2):245-254.

Rohfl, F.J. 1992. NTSYS-pc Numerical taxonomy and multivariate analysis systems. Versión 1.70. Exeter Publishing. New York, USA. 250 p.

Sousa, J; Meletti, L. 1997. Maracujá: espécies, variedades, cultivo. FEALQ. São Paulo, Brasil. 179 p.

Tang, T; Zhong, Y; Jian, S; Shi, S. 2003. Genetic diversity of Hibiscus tiliaceus (Malvaceae) in China assessed using AFLP markers. Annals of Botany 92:409-414.

Wagara, I; Mwang'Ombe, A; Kimenju, J; Buruchara, R; Jamnadass, R; Majuwa, P. 2004. Genetic diversity of Phaeoisariopsis griseola in Kenya as revealed by AFLP and group-specific primers. Journal of Phytopathology 152:235- 242.

Yeh, FY; Boyle, R; Ye, T; Mao,Z. 1997. POPGENE, the user friendly shareware for population genetic analysis. Versión 1.32. Molecular Biology and Biotechnology Centre, University of Alberta, Alberta, USA. 29 p. 